\title{
ASTRÁGALO E OSTEODERMOS DE GLYPTOTHERIUM OSBORN, 1903 DO PLEISTOCENO SUPERIOR DE PERNAMBUCO, NORDESTE DO BRASIL
}

\author{
LUANA CARDOSO DE ANDRADE \\ Programa de Pós-Graduação em Geociências, Laboratório de Paleontologia, Universidade Federal de Pernambuco, Av. Prof. \\ Moraes Rego, 1235, Cidade Universitária, 50670-901, Recife, Pernambuco, Brasil. \\ luanacardosodeandrade@gmail.com \\ ÉDISON VICENTE OLIVEIRA \\ Departamento de Geologia, Laboratório de Paleontologia, Universidade Federal de Pernambuco, Av. Prof. Moraes Rego, \\ 1235, Cidade Universitária, 50670-901, Recife, Pernambuco, Brasil.vicenteedi@gmail.com \\ VANDERLEI MANIESI \\ Departamento de Geografia, Grupo de Pesquisa Geociências, Universidade Federal de Rondônia, Campus - BR 364, Km \\ 9,5, 76801-059, Porto Velho, Rondônia, Brasil.maniesi@unir.br

\section{SÔNIA HATSUE TATUMI} \\ Departamento de Ciências do Mar, Universidade Federal de São Paulo, Rua Dr. Carvalho de Mendonça, 144, 11070-100, \\ Santos, SP, Brasil.sonia.tatumi@gmail.com
}

\begin{abstract}
Astragalus and osteoderms of Glyptotherium Osborn, 1903 from the Upper Pleistocene of Pernambuco, Northeast of Brazil. In Brazil, Glyptotherium findings occur mainly in the Northeastern region. In the northeast, Brazilian Intertropical Region (BIR), there are records of Glyptotherium of the Upper Pleistocene, mainly in the State of Pernambuco. This work reports new findings of Glyptotherium in a tank deposit from the Upper Pleistocene of Pernambuco, corresponding to isolated osteoderms and an astragalus which approaches in detail to North American forms. This record expands both the distribution of Glyptotherium to the BIR, and the data regarding the paleobiogeographic relations among the faunas of megamammals of the North of South America, Central America, and the South of North America during the Late Pleistocene.
\end{abstract}

Keywords: Glyptotherium, taxonomy, Late Pleistocene, tank deposit, Brazil.

RESUMO - No Brasil, os achados de Glyptotherium ocorrem, principalmente, na região Nordeste. No Nordeste, Região Intertropical Brasileira (RIB), há registros de Glyptotherium do Pleistoceno Superior, principalmente no Estado de Pernambuco. Este trabalho reporta novos achados de Glyptotherium em um depósito de tanque do Pleistoceno de Pernambuco, correspondendo a osteodermos isolados e um astrágalo que se aproxima em detalhe as formas norte-americanas. Esse registro amplia tanto a distribuição de Glyptotherium para a RIB quanto os dados referentes às relações paleobiogeográficas entre as faunas de megamamíferos do norte da América do Sul, da América Central e do sul da América do Norte durante o Neopleistoceno.

Palavras-chave: Glyptotherium, taxonomia, Pleistoceno Superior, depósito de tanque, Brasil.

\section{INTRODUÇÃO}

Os extintos Glyptodontinae são um dos grupos de mamíferos Xenarthra mais registrados para a América do Sul, com grande diversidade durante o Cenozoico (McKenna \& Bell, 1997; Zurita et al., 2012). O conhecimento da distribuição paleobiogeográfica dos Glyptodontinae pleistocênicos na América do Sul em especial, tem sido reformulado com base em revisões recentes (e.g. Carlini et al., 2008; Oliveira et al., 2010; Zurita et al., 2012).
Um dos casos mais notáveis tem sido a identificação do gênero norte-americano Glyptotherium na América do Sul, adicionando um novo elemento ao Grande Intercâmbio Faunístico Americano - GABI. Os registros abrangem, além da Venezuela, parte da região Nordeste do Brasil (Zurita et al., 2012), pertencente à Região Intertropical Brasileira (sensu Cartelle, 1999).

Os materiais atribuídos a Glyptotherium no nordeste do Brasil compreendem, até o momento, osteodermos isolados oriundos de Conceição das Creoulas e Fazenda 
Nova, Pernambuco, materiais do esqueleto apendicular e osteodermos coletados em cavernas calcárias de Lajedo da Escada, Rio Grande do Norte (Oliveira et al., 2010; Barbosa et al., 2014) e osteodermos isolados de um depósito de tanque no munícipio do Congo, Paraíba (Andrade et al., 2016, 2017).

Os registros de Glyptotherium no nordeste do Brasil ainda são escassos, quando comparados a outros táxons coletados em depósitos de tanques, incluindo Pernambuco (e.g. Eremotherium laurillardi) (Araújo Júnior et al., 2013). Neste trabalho, registram-se novos materiais de Glyptotherium provenientes de um depósito de tanque do Pleistoceno Superior do Estado de Pernambuco, e elucidam-se suas implicações paleobiogeográficas.

\section{LOCALIZAÇÃO GEOGRÁFICA E ESTRATIGRÁFICA}

O material estudado foi coletado no tanque Zabelê ( $8^{\circ} 43^{\prime} 15^{\prime \prime} \mathrm{S}, 36^{\circ} 38^{\prime} 20^{\prime \prime} \mathrm{W}$, altitude $885 \mathrm{~m}$ ), localizado na microrregião do Vale do Ipojuca, mesorregião do agreste pernambucano, Nordeste do Brasil. Partindo-se da capital Recife, o acesso ao tanque pode ser feito através da BR-232 sentido Caruaru e, em seguida, pela PE-193 (Figura 1).

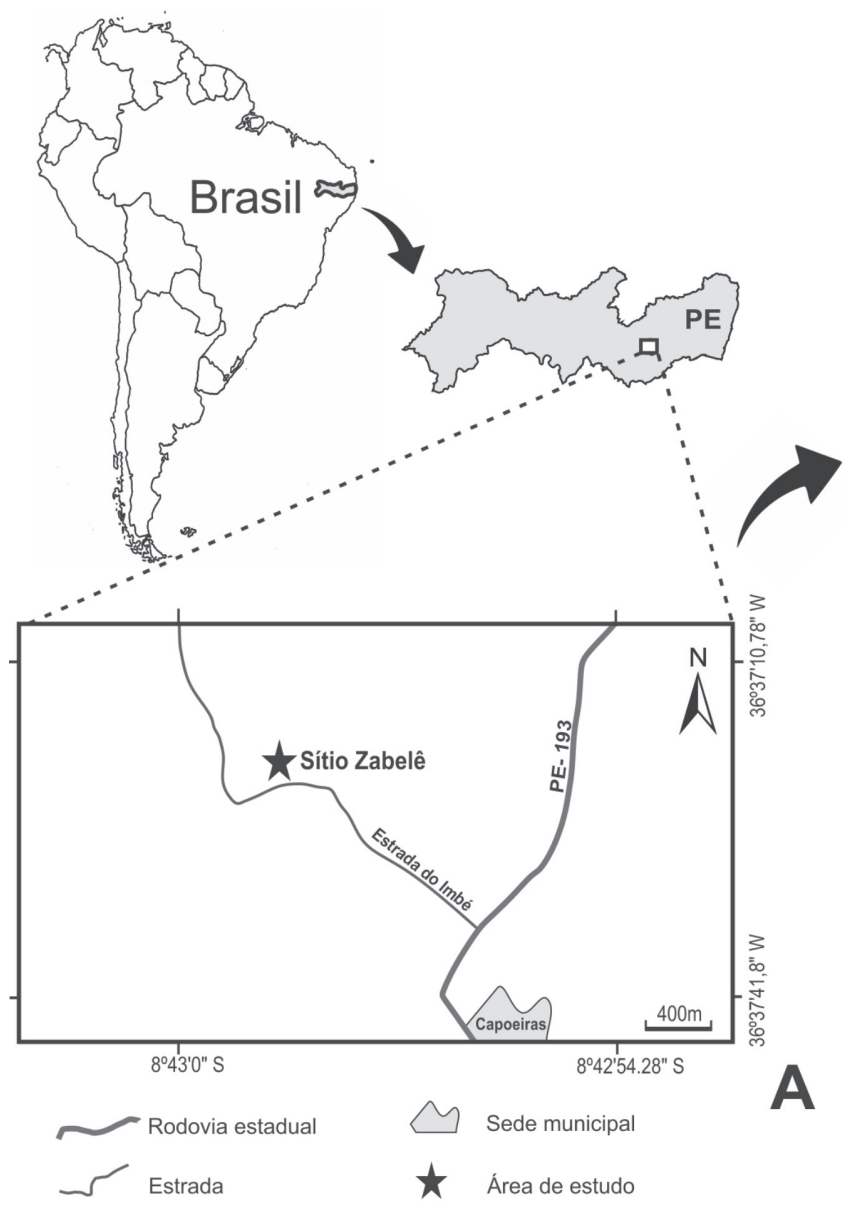

O tanque Zabelê apresenta um formato elíptico com cerca de $250 \mathrm{~cm}$ de profundidade. O material descrito foi encontrado juntamente com restos esqueletais da megamamíferos atribuídos a Eremotherium laurillardi, Notiomastodon platensis, Toxodon platensis, Glyptotherium sp., e Protocyon troglodites.

Geologicamente, o tanque está inserido na Província Borborema, constituída pelos litotipos mesoproterozoicos dos Complexos Cabrobó e Belém do São Francisco e pelos granitoides neoproterozoicos da Suíte Intrusiva Leucocrática Peraluminosa (CPRM, 2005). O embasamento rochoso do tanque é composto por granitoides neoproterozoicos, idade de 580 M.a. (Brito Neves et al., 2000), que intemperizam para um material argilo-síltico-arenoso (CPRM, 2005).

O preenchimento sedimentar do depósito de tanque consiste em quatro camadas distintas (da base ao topo): Camada I. De $50 \mathrm{~cm}$ de espessura constituída por areia média a grossa com matriz composta de argila, silte e litoclastos (retrabalhada do embasamento). Camada II. De 30 a $40 \mathrm{~cm}$ de cascalho com seixos subangulosos de quartzo que variam de 25 a $40 \mathrm{~mm}$ no eixo principal. A matriz é predominantemente de areia com restos de mamíferos e não apresentam orientação preferencial. Os sedimentos da porção intermediária desta Camada II foram datados em 41.000

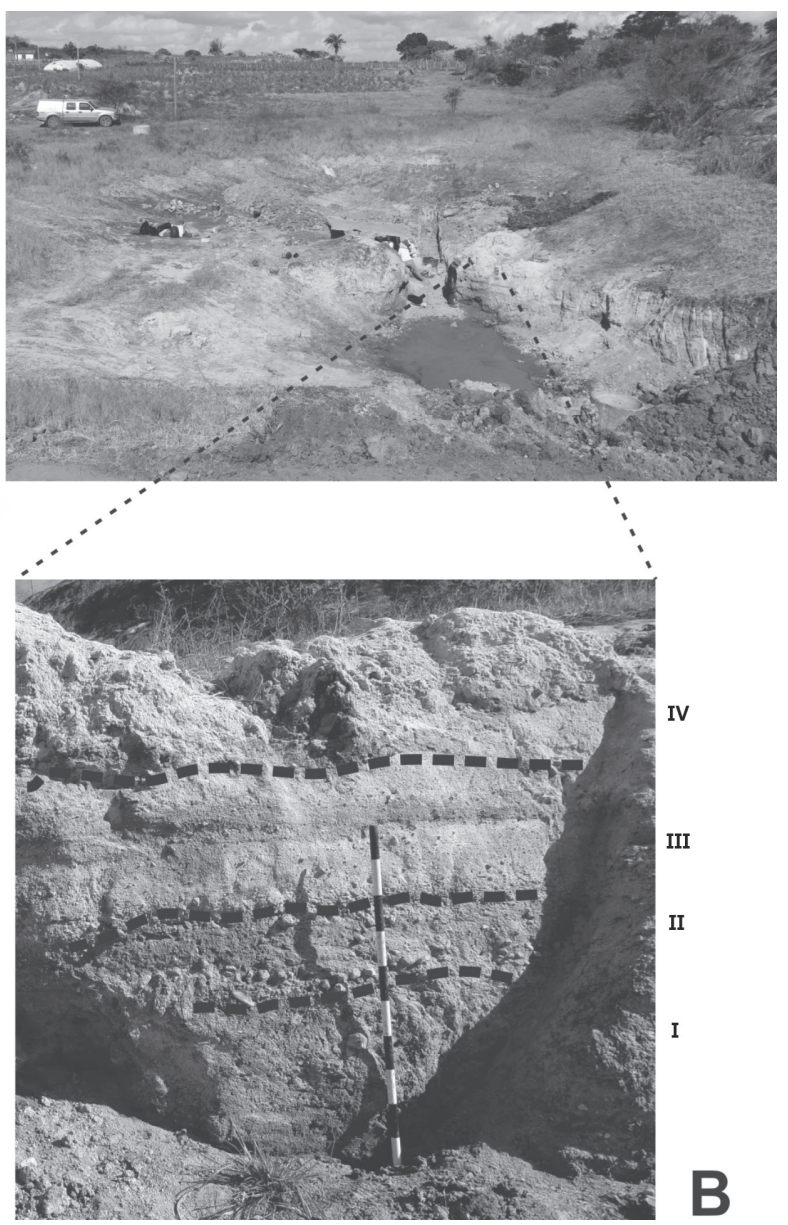

Figura 1. A, localização do tanque Zabelê, Município de Capoeiras, Estado de Pernambuco, Brasil. B, detalhe do preenchimento sedimentar do tanque.

Figure 1. A, location of Zabelê tank, Municipality of Capoeiras, State of Pernambuco, Brazil. B, detail of the sedimentary infilling of the tank. 
A.P. \pm 2.100 AP (Neopleistoceno), com base no método de Luminescência Opticamente Estimulada (LOE), realizada pelo Laboratório Espectrometria Gama e Luminescência, do Instituto de Geociências da Universidade de São Paulo (USP). Os fósseis ocorrem acima e abaixo dos sedimentos datados. Camada III. De $50 \mathrm{~cm}$ de espessura ligeiramente bioturbada, constituída por sedimentos arenosos com grânulos e seixos subangulares dispersos. Camada IV. De $110 \mathrm{~cm}$ de espessura de sedimentos enlameados ricos em conteúdo orgânico. Considerando apenas os aspectos texturais da camada fossilífera, sua origem é relacionada a um sistema aluvial ou fluvial.

\section{MATERIAL E MÉTODOS}

O material estudado é parte da coleção de macrofósseis do Laboratório de Paleontologia do Departamento de Geologia (DGEO) da Universidade Federal de Pernambuco (UFPE), em Recife. A abreviatura utilizada para identificar a coleção científica é DGEO-CTG-UFPE. Os materiais estudados foram comparados com osteodermos de Glyptotherium sp. e astrágalos de Panochthus sp. e Hoplophorus euphractus da Coleção de referência do DGEO-CTG-UFPE e com figurações e descrições na literatura específica: Owen (1841), Winge (1915), Gillette \& Ray (1981), Porpino \& Bergqvist (2002) e Porpino et al. (2014).

A taxonomia e a nomenclatura usadas seguem Carlini et al. (2008), Oliveira et al. (2009, 2010), Zurita et al. (2012) e Porpino et al. (2010). As estruturas da ornamentação externa dos osteodermos consideradas neste trabalho estão ilustradas na Figura 2.

Nos osteodermos estudados foram realizadas medições em $\mathrm{mm}$. Para melhor visualização da morfologia dos osteodermos, como, por exemplo, a ocorrência de orifícios com pequeno diâmetro (1-1,5mm), foi utilizada a lupa estereoscópica com as objetivas de $0,5 \mathrm{x}$ a $1,5 \mathrm{x}$ e aumento de até $2 \mathrm{x}$.

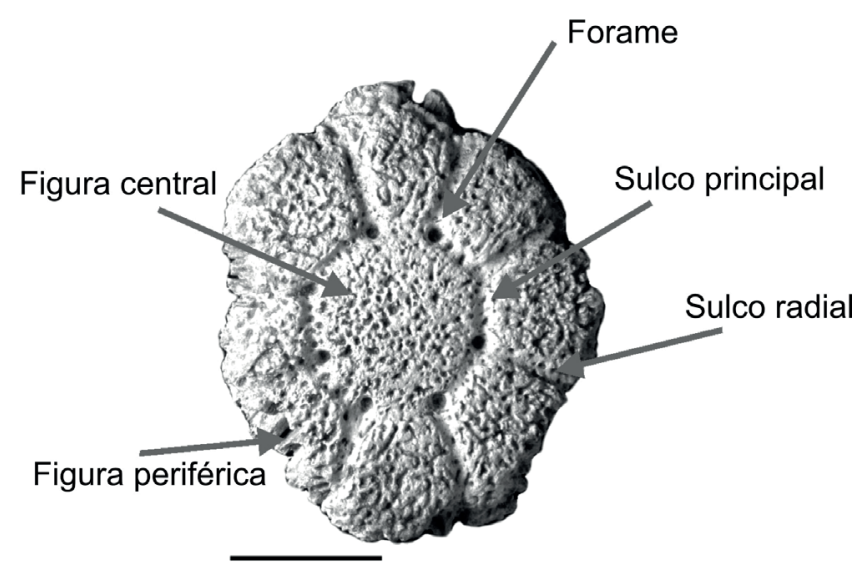

Figura 2. Nomenclatura usada para a morfologia dos osteodermos (em vista dorsal) em Glyptotherium sp. Modificado de Oliveira et al. (2010). Escala $=2 \mathrm{~cm}$.

Figure 2. Nomenclature used for the morphology of osteoderms (in dorsal view) in Glyptotherium sp. Modified from Oliveira et al. (2010). Scale bar $=2 \mathrm{~cm}$.

\section{PALEONTOLOGIA SISTEMÁTICA}

\author{
XENARTHRA Cope, 1889 \\ CINGULATA Illiger, 1811 \\ GLYPTODONTIDAE Burmeister, 1879 \\ GLYPTODONTINAE Gray, 1869 \\ (nom. transl. Trouessart, 1898) \\ Glyptotherium Osborn, 1903 \\ (Figuras 3-6; Tabelas 1-2)
}

Materiais referidos. Osteodermos isolados da região dorsal (DGEO-CTG-UFPE 7814, 7815, 7816, 7817, 7819) e do anel caudal (DGEO-CTG-UFPE 7820); astrágalo direito (DGEOCTG-UFPE 7978).

Descrição. Osteodermos: os pertencentes à região dorsal da carapaça apresentam formato hexagonal, superfície externa uniformemente perfurada, configurando orifícios com diâmetro entre 1 e $1,5 \mathrm{~mm}$, resultando em um aspecto rugoso. Os forames, com diâmetro entre 1,5 a 2,5 mm, estão situados nas intersecções dos sulcos radiais com o principal ou próximos dessas (DGEO-CTG-UFPE 7814, 7815 e 7817). A figura central é plana, variando de circular a subcircular e as figuras periféricas variam de seis a oito, com formato poligonal e com tamanho similar (quando preservadas). $\mathrm{O}$ espécime DGEO-CTG-UFPE 7817, região dorsal da carapaça, é composto por dois osteodermos fusionados (Figura 3A, Tabela 1). Já o espécime DGEO-CTG-UFPE 7819 preserva o formato hexagonal e a dimensão e morfologia de osteodermos da região dorsal da carapaça, porém, devido ao forte grau de abrasão, não exibe figuras centrais e periféricas na superfície externa (Figura 3C).

O espécime DGEO-CTG-UFPE 7816 possui a superfície ventral côncava (vista lateral). Em vista ventral, os forames situados na porção central estão ausentes, possivelmente devido ao grau de abrasão (Figuras 4A', A"). A porção central da superfície externa apresenta ausência da figura central e sulcos radiais distintos (Figura 4A). Possui, ainda, menor espessura e tamanho (Tabela 1). Nas Figuras 4A' e 4A" é possível notar, apesar do desgaste, uma figura principal mais elevada, o que também pode indicar característica de espécimes juvenis (Zurita et al., 2011; Gillette et al., 2015).

$\mathrm{O}$ osteodermo da região do anel caudal, possivelmente da porção mais posterior (DGEO-CTG-UFPE 7920) (Figura 4B), é subquadrangular (vista externa) com superfície externa muito pontuada e rugosa, possuindo uma proeminência cônica moderadamente desenvolvida e maior espessura em relação aos demais osteodermos. Encontra-se fraturado na borda lateral (vista lateral) (Figura 4B). Os osteodermos da região mais posterior do anel caudal são marcadamente cônicos (Figura 4B) (Gillette \& Ray, 1981).

Astrágalo: comparativamente, o ástragalo apresenta a tróclea assimétrica, com projeção mais proximal do que lateral, assim como em Glyptodon (Owen, 1841), mas difere de Panochthus sp. e Hoplophorus euphractus onde ela é fortemente assimétrica, com a crista lateral mais desenvolvida (Figuras 6A-D). O sulco entre as cristas da tróclea astragalar 

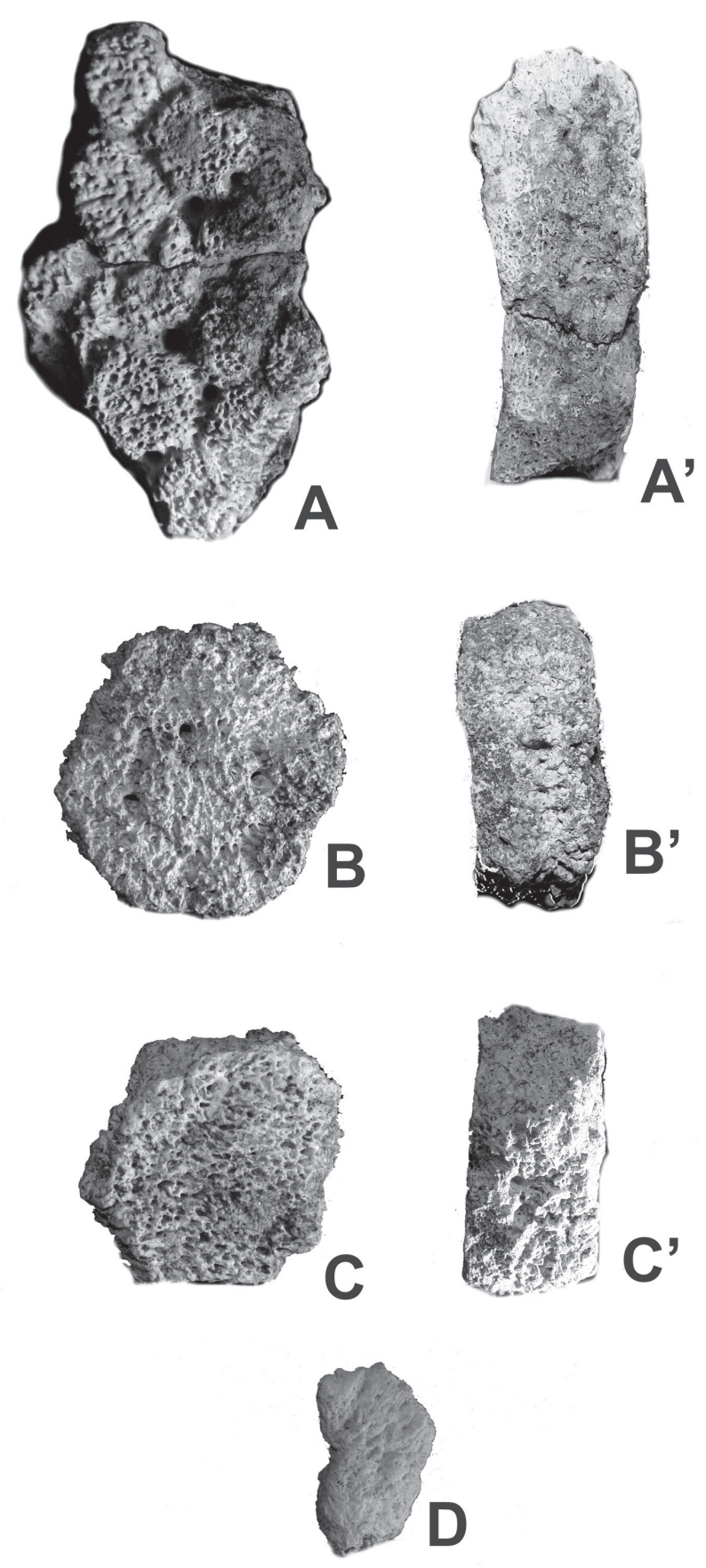

Figura 3. Osteodermos isolados da carapaça de Glyptotherium sp. A, DGEOCTG-UFPE 7817, vista externa; A', vista lateral. B, DGEO-CTG-UFPE 7814, vista externa; B', vista lateral. C, DGEO-CTG-UFPE 7819, vista externa; C', vista lateral. D, DGEO-CTG-UFPE 7815, vista externa. Escala $=5 \mathrm{~cm}$.

Figure 3. Isolated osteoderms of carapace of Glyptotherium sp. A, DGEOCTG-UFPE 7817, external view; A', lateral view. B, DGEO-CTG-UFPE 7814, external view; B', lateral view. C, DGEO-CTG-UFPE 7819, external view; C', lateral view. D, DGEO-CTG-UFPE 7815, external view. Scale bar $=5 \mathrm{~cm}$.

é mais raso e estreito, assim como em Glyptodon, sendo menos marcado e desenvolvido do que em Panochthus sp. e H. euphractus (Figuras 6A-D).
Tabela 1. Medidas (mm) de osteodermos de Glyptotherium sp. da Coleção DGEO-CTG-UFPE provenientes do tanque Zabelê, Município de Capoeiras, Pernambuco, Brasil. Abreviações: *, espécime fraturado, medidas comprometidas; 1, largura; 2, comprimento anteroposterior; $\mathbf{3}$, espessura; $\mathbf{4}$, comprimento da figura central; 5 , largura da figura central.

Table 1. Measurements ( $\mathrm{mm}$ ) of osteoderms of Glyptotherium sp. of the DGEO-CTG-UFPE Collection from Zabelê tank, Capoeiras Municipality, Pernambuco, Brazil. Abbreviations: *, fractured specimen, estimated measurements; 1, width; 2 , anteroposterior length; $\mathbf{3}$, thickness; $\mathbf{4}$, length of central figure; $\mathbf{5}$, width of central figure.

\begin{tabular}{cccccc}
\hline Glyptotherium sp. & \multicolumn{5}{c}{ Medidas } \\
\hline Espécimes & 1 & 2 & 3 & 4 & 5 \\
DGEO-CTG-UFPE 7814 & 44 & 44,5 & 18 & 18 & 19,5 \\
DGEO-CTG-UFPE 7815* & 29 & 40 & 16 & - & - \\
DGEO-CTG-UFPE 7816 & 30 & 40 & 16 & - & - \\
DGEO-CTG-UFPE 7817** & 42 & 73 & 23 & 15 & 25,5 \\
DGEO-CTG-UFPE 7819 & 42 & 40 & 20 & - & - \\
DGEO-CTG-UFPE 7820 & 46 & 53 & 33 & - & - \\
\hline
\end{tabular}

Tabela 2. Medidas (mm) dos astrágalos de Glyptotherium sp., Panochthus sp. e Hoplophorus euphractus, provenientes da Coleção DGEO-CTG-UFPE. Abreviações: 1, comprimento máximo; 2, altura máxima; 3, comprimento da faceta sustentacular; $\mathbf{4}$, altura da faceta sustentacular; $\mathbf{5}$, comprimento da faceta ectal; $\mathbf{6}$, altura da faceta ectal.

Table 2. Measurements ( $\mathrm{mm}$ ) of the astragalus of Glyptotherium sp., Panochthus sp., and Hoplophorus euphractus, from DGEO-CTG-UFPE Collection. Abbreviations: 1, maximum lenght; 2, maximum height; 3, length of the sustentacular facet; $\mathbf{4}$, height of the sustentacular facet; $\mathbf{5}$, length of the ectal facet; $\mathbf{6}$, height of the ectal facet.

\begin{tabular}{|c|c|c|c|c|c|c|}
\hline \multirow{2}{*}{ Espécimes } & \multicolumn{6}{|c|}{ Medidas } \\
\hline & 1 & 2 & 3 & 4 & 5 & 6 \\
\hline $\begin{array}{c}\text { Panochthus sp. } \\
\text { (DGEO-CTG-UFPE 6513) }\end{array}$ & 111 & 84 & 27 & 43 & 42 & 49 \\
\hline $\begin{array}{c}\text { H. euphractus } \\
\text { (DGEO-CTG-UFPE 6512) }\end{array}$ & 75 & 58,5 & 20,2 & 32,5 & 34 & 41,2 \\
\hline $\begin{array}{c}\text { Glyptotherium sp. } \\
\text { (DGEO-CTG-UFPE 7978) }\end{array}$ & 90 & 72 & 34 & 40 & 34 & 39 \\
\hline
\end{tabular}

As facetas articulares para o calcâneo diferem. A faceta ectal em DGEO-CTG-UFPE 7978 é mais alongada proximodistalmente, subretangular, com contorno arredondado, sendo que em Panochthus sp. e Hoplophorus euphractus é marcadamente triangular (Figuras 6E-H). Já a faceta sustentacular é côncava e irregular, com formato elipsoide, mais semelhante a Glyptodon e diferindo de Panochthus sp. e $H$. euphractus, que apresentam uma faceta plana, elipsoide, porém elevada (Figuras 6E-H). O sulcus tali é menos profundo e mais estreito em DGEO-CTG-UFPE 7978 do que em Panochthus sp. e H. euphractus (Figuras $6 \mathrm{E}-\mathrm{H})$. A tuberosidade navicular ocorre proximalmente junto à borda dorsal da faceta navicular, tendo o formato cilíndrico semelhante à Glyptotherium texanum (Figuras 5 e 6). 


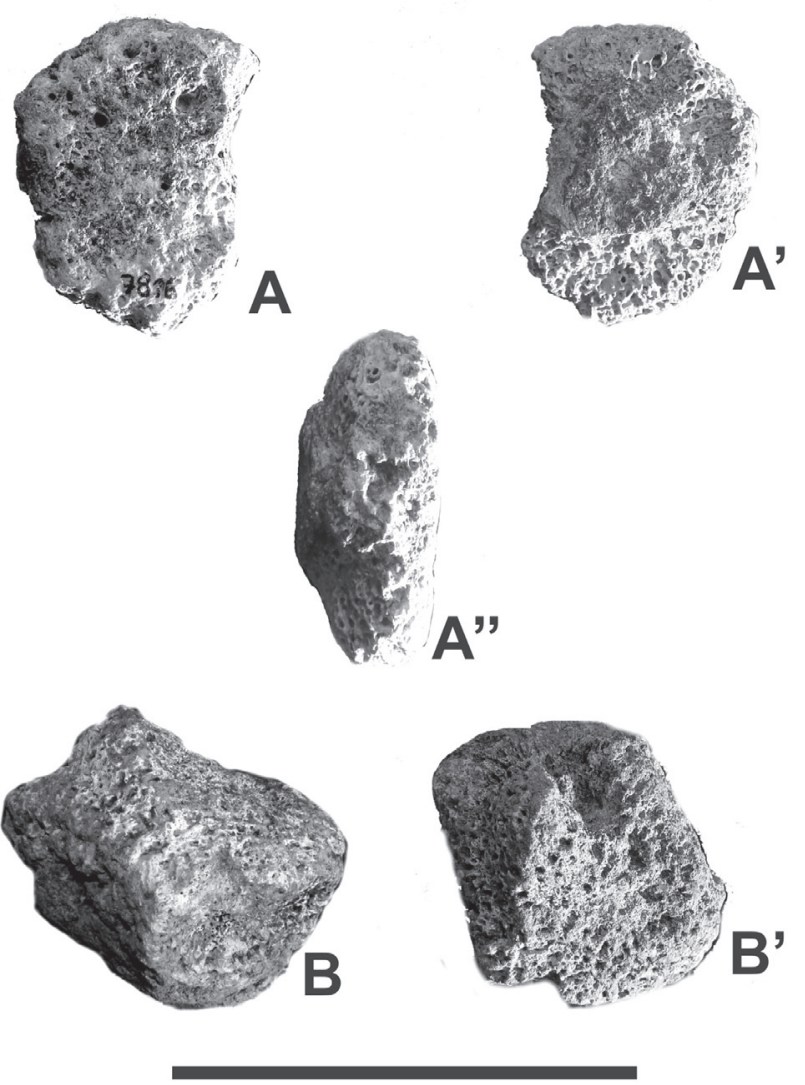

Figura 4. Osteodermos isolados da carapaça de Glyptotherium sp. A, DGEO-CTG-UFPE 7816, região dorsal (vista externa); A', vista anterior; A", vista lateral. B, DGEO-CTG-UFPE 7820, anel caudal (vista lateral); B', vista externa. Escala $=5 \mathrm{~cm}$.

Figure 4. Isolated osteoderms of carapace of Glyptotherium sp. A, DGEO-CTG-UFPE 7816, dorsal region (external view); A', anterior view; A", lateral view. B. DGEO-CTG-UFPE 7820, caudal ring (lateral view); B', external view. Scale bar $=5 \mathrm{~cm}$.

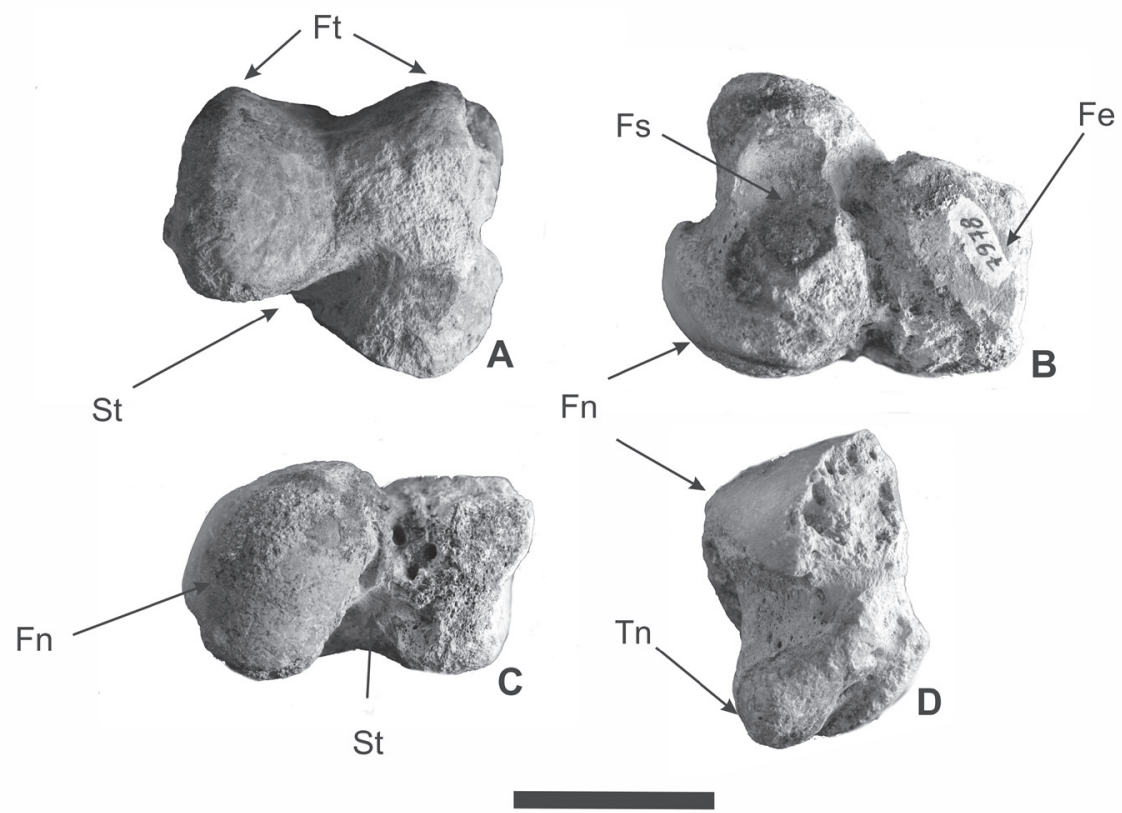

Figura 5. Ástragalo direito de Glyptotherium sp. DGEO-CTG-UFPE 7978, proveniente do tanque Zabelê, Município de Capoeiras, Pernambuco, Brasil. A, vista dorsal; B, vista plantar; C, vista distal; D, vista cranial. Abreviações: Ft, faceta troclear; St, sulcus tali; Fe, faceta ectal; Fs, faceta sustentacular; Fn, faceta para o navicular; Tn, tuberosidade para o navicular. Escala $=7 \mathrm{~cm}$.

Figure 5. Right astragalus of Glyptotherium sp. DGEO-CTG-UFPE 7978 from Zabelê tank, Capoeiras Municipality, Pernambuco, Brazil. A, dorsal view; B, plantar view; C, distal view; D, cranial view. Abbreviations: Ft, troclear facet; St, sulcus tali; Fe, ectal facet; Fs, sustentacular facet; Fn, facet for the navicular; Tn, tuberosity for the navicular. Scale bar $=7 \mathrm{~cm}$. 

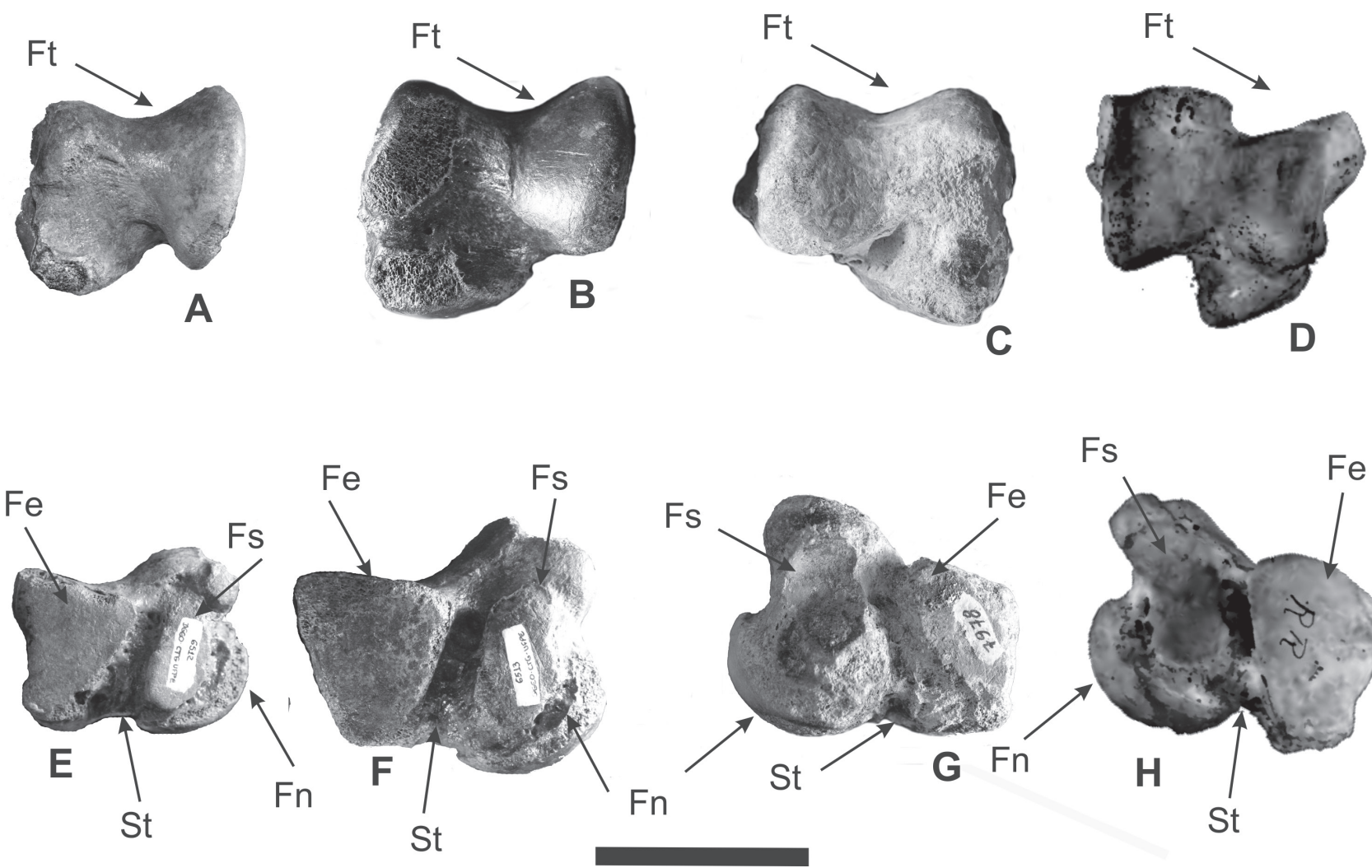

Fs

Figura 6. Comparação dos astrágalos. Hoplophorus euphractus (esq.) (DGEO-CTG-UFPE 6512), localidade Lagoa do Tanque, Pernambuco, Brasil (A, vista proximal; E, vista plantar). Panochthus sp. (esq.) (DGEO-CTG-UFPE 6513), localidade Lagoa do Tanque, Pernambuco, Brasil (B, vista proximal; F, vista plantar). Glyptotherium sp. (DGEO-CTG-UFPE 7978), tanque Zabelê, Pernambuco, Brasil (C, vista proximal; G, vista plantar). Glyptotherium texanum (dir.) (AM 95737), localidade “Tusker local fauna”, Graham County, Arizona (modificado de Gillette \& Ray, 1981) (D. vista proximal; H, vista plantar). Abreviações: Ft, faceta troclear; St, sulcus tali; $\mathbf{F e}$, faceta ectal; Fs, faceta sustentacular; $\mathbf{F n}$, faceta para o navicular; Tn, tuberosidade para o navicular. Escala $=7 \mathrm{~cm}$.

Figure 6. Comparison of astragalus. Hoplophorus euphractus (left) (DGEO-CTG-UFPE 6512), Lagoa do Tanque locality, Pernambuco, Brazil (A. proximal view; E, plantar view). Panochthus sp. (left) (DGEO-CTG-UFPE 6513), Lagoa do Tanque locality, Pernambuco, Brazil (B, proximal view; F, plantar view). Glyptotherium sp. (DGEO-CTG-UFPE 7978), Zabelê tank, Pernambuco, Brazil (C, proximal view; G, plantar view). Glyptotherium texanum (right) (AM 95737), "Tusker local fauna" locality, Graham County, Arizona (modified from Gillette \& Ray, 1981) (D, proximal view; H, plantar view). Abbreviations: $\mathbf{F t}$, troclear facet; St, sulcus tali; Fe, ectal facet; Fs, sustentacular facet; Fn, facet for the navicular; Tn, tuberosity for the navicular. Scale bar $=7 \mathrm{~cm}$.

\section{DISCUSSÃO}

Os osteodermos estudados pertencem a Glyptotherium sp. por apresentar a combinação de características propostas por Oliveira et al. (2009, 2010): figura central plana, de maior diâmetro, superfície externa muito perfurada, aparência rugosa, sulcos radiais e principais mais rasos e um número de oito ou nove figuras periféricas. Trata-se de um padrão de ornamentação tido como primitivo (Carlini et al., 2008). Os caracteres morfológicos aqui discutidos diferem de Glyptodon, pois neste gênero os osteodermos possuem superfície externa mais lisa, sulco principal e sulcos radiais mais profundos e um número menor de figuras periféricas (Ameghino, 1889; Castellanos, 1953; Soibelzon et al., 2006; Oliveira et al., 2009, 2010). A morfologia dos osteodermos aqui descrita é semelhante àquela encontrada em outras regiões da RIB (e.g. Lajedo da Escada, Rio Grande do Norte, Oliveira et al., 2010) e da região de Lagoa Santa, Minas Gerais, atribuídos à Glyptotherium sp. (Oliveira et al., 2009; 2010).
O astrágalo é atribuído a Glyptotherium sp. por possuir as características descritas por Gillete \& Ray (1981) como, por exemplo, as facetas articulares pequenas, sendo a faceta sustentacular côncava, irregular e com forma elipsoide e a faceta ectal com formato subretangular. DGEO-CTG-UFPE 7978 apresenta morfologia semelhante à de Glyptodontinae, em especial a G. texanum (Gillete \& Ray, 1981), com pequenas diferenças como, por exemplo, a faceta sustentacular, que é mais desenvolvida na espécie norte-americana. Entretanto, essas diferenças também podem ter sido acentuadas pelo grau de abrasão do espécime aqui referido. Como aqui se trata de um único espécime, inferências morfológicas comparativas tornam-se limitadas.

Em relação aos Hoplophorinae, há diferenças significativas principalmente no que diz respeito às facetas ectais (triangulares) e sustentacular (mais elevada) e ao sulcus tali (mais profundo) nesses indivíduos do que em DGEOCTG-UFPE 7978. Os espécimes diferem também, em relação às dimensões, pois quando comparado ao espécime 
DGEO-CTG-UFPE 7978, Panochthus sp. apresenta maiores dimensões enquanto que o Hoplophorus euphractus possui menores dimensões.

Além disso, a distribuição paleobiogeográfica corrobora a atribuição a Glyptotherium, já que esses indivíduos ocorrem na região Nordeste do Brasil (Carlini et al., 2008; Zurita et al., 2012), diferentemente de Glyptodon que é encontrado na região sul do Brasil (e.g. Oliveira et al., 2010).

As ocorrências de Glyptotherium no Pleistoceno Superior da América do Sul $(41.000 \pm 2.100$ AP, LOE-camada fossilífera), representam o resultado de eventos de migrações massivas da América do Norte ou vice-versa durante o Último Máximo Glacial (UMG) ou um pouco antes desse evento, por meio de possíveis corredores biogeográficos em áreas de planícies, já que o nível do mar estava abaixo do atual (Carlini et al., 2008; Woodburne, 2010).

Esse e outros achados de Glyptotherium na RIB mostram relações paleobiogeográficas mais complexas entre as faunas de mamíferos do norte da América do Sul, da América Central e do sul da América do Norte durante o Neopleistoceno. Além disso, é possível inferir condições climáticas e ecológicas similares para essas regiões (Scillato-Yané et al., 2005; Carlini et al., 2008).

\section{CONCLUSÃO}

Os osteodermos e o astrágalo coletados no tanque Zabelê em Capoeiras, Pernambuco, pertencem a Glyptotherium sp. Os osteodermos apresentam a combinação de caracteres tida para o gênero. Já o astrágalo é morfologicamente distinto de Hoplophorinae, assemelhando-se à Glyptodontinae, exibindo caracteres potencialmente derivados presentes em espécies de Glyptotherium norte-americanas, como as facetas articulares pequenas e curvadas, sendo a faceta ectal côncava, irregular e com forma elipsoide e a faceta sustentacular com formato subretangular.

Os novos achados de Glyptotherium para Pernambuco aliados aos dados geocronológicos $(41.000 \mathrm{AP} \pm 2.100 \mathrm{AP})$ corroboram o intervalo temporal dos tanques da RIB e suas relações paleobiogeográficas no Neopleistoceno entre os mamíferos da RIB e da América do Sul, da América Central e do Sul da América do Norte.

\section{AGRADECIMENTOS}

A Coordenação de Aperfeiçoamento de Pessoal de Nível Superior/CAPES pela bolsa concedida à primeira autora. A equipe do Laboratório de Paleontologia-PALEOLAB da UFPE, pelo auxílio nos trabalhos de campo. À Prefeitura e à Secretaria de Cultura do Município de Capoeiras (PE) pelo apoio logístico e disponibilidade de maquinário utilizado nas escavações em campo. Os autores agradecem a A.E. Zurita (Universidad Nacional del Nordeste/UNNE) e a K.A. Porpino (Universidade do Estado do Rio Grande do Norte/UERN) pelas valiosas contribuições ao manuscrito.

\section{REFERÊNCIAS}

Ameghino, F. 1889. Contribución al conocimiento de los mamíferos fósiles de la República Argentina. Actas de la Academia Nacional de Ciencias, 6:1-1027.

Andrade, L.C.; Oliveira, E.V. \& Souza, B.I. 2016. Novos achados de Xenarthra em um depósito de tanque do Pleistoceno da Paraíba, nordeste do Brasil. In: REUNI ̃̃O DA SBPV- PALEO NORDESTE, 2016. Livro de Resumos, Mossoró, SBP, p. 42.

Andrade, L.C.; Oliveira, E.V. \& Souza, B.I. 2017. Novos achados de Xenarthra em um depósito de tanque do Pleistoceno da Paraíba, nordeste do Brasil. Revista de Geologia UFC, 30:33-46.

Araújo-Júnior, H.I.; Porpino, K.O. \& Bergqvist, L.P. 2013. Taphonomic analysis of a Late Pleistocene vertebrate accumulation from Lage Grande Paleontological Site, Pernambuco State, Northeastern Brazil: new remarks on preservational aspects of tank deposits. Quaternary International, 317:88-101. doi:10.1016/j.quaint.2013.08.016

Barbosa, F.H.S.; Porpino, K.O.; Fragoso, A.B.L. \& Oliveira, E.V. 2014. Arthritis in a Glyptodont (Mammalia, Xenarthra, Cingulata). PLoS ONE, 9:e88646. doi:10.1371/journal. pone. 0088646

Brito Neves, B.B.; Santos, E.J. \& Van Schmus, W.R. 2000. Tectonic history of the Borborema Province. In: U. Cordani (ed.) Tectonic evolution of South America, p. 151-182.

Carlini, A.A.; Zurita, A.E. \& Aguilera, O.A. 2008. North American glyptodontines (Xenarthra, Mammalia) in the Upper Pleistocene of northern South America. Palaontologische Zeitschrift, 82:125-138. doi:10.1007/BF02988404

Cartelle, C. 1999. Pleistocene mammals of the Cerrado and Caatinga of Brazil. In: J.B. Eisenberg \& K.H. Redford (eds.) Mammals of the neotropics: the central tropics, University of Chicago Press, p. 27-46.

Castellanos, A. 1953. Anotações e retificações ao gênero Glyptodon Owen e à subfamília Glyptodontinae. Anais da Academia Brasileira de Ciências, 25:391-410.

CPRM - Serviço Geológico do Brasil. 2005. Projeto cadastro de fontes de abastecimento por água subterrânea. Diagnóstico do municipio de Capoeiras, estado de Pernambuco. Recife, 11 p.

Gillette, D.D.; Carranza-Castañeda, O.; White Jr., R.S.; Morgan, G.S.; Thrasher, L.C.; McCord, R. \& McCullough, G. 2015. Ontogeny and sexual dimorphism of Glyptotherium texanum (Xenarthra, Cingulata) from the Pliocene and Pleistocene (Blancan and Irvingtonian NALMA) of Arizona, New Mexico, and Mexico. Journal of Mammalian Evolution, 23:133-154. doi:10.1007/s10914-015-9309-6

Gillette, D.D. \& Ray, C.E. 1981. Glyptodonts of North America. Washington, D.C., Smithsonian Institution Press, 255 p. (Contributions to Paleobiology 40).

McKenna, M.C. \& Bell, S.K. 1997. Classification of mammals. Above the species level. New York, Columbia University Press, $631 \mathrm{p}$.

Oliveira, E.V.; Barreto, A.M.F. \& Alves, R.A. 2009. Aspectos sistemáticos, paleobiogeográficos e paleoclimáticos dos mamíferos quaternários de Fazenda Nova, PE, nordeste do Brasil. GAEA-Journal of Geoscience, 5:75-85. doi:10.4013/ gaea.2009.52.04

Oliveira, E.V.; Porpino, K.O. \& Barreto, A.M.F. 2010. On the presence of Glyptotherium in the Late Pleistocene of Northeastern Brazil, and the status of "Glyptodon" and "Chlamydotherium". Paleobiogeographic implications. Neues Jahrbuch für Geologie und Paläontologie, 258:353-363. doi:10.1127/0077-7749/2010/0116 
Owen, R. 1841.VI. - Description of a tooth and part of the skeleton of the Glyptodon clavipes, a large quadruped of the Edentate order. Transactions of the Geological Society of London, 6:81-106. doi:10.1144/transgslb.6.1.81

Porpino, K.O. \& Bergqvist, L.P. 2002. Novos achados de Panochthus (Mammalia, Cingulata, Glyptodontoidea) no nordeste do Brasil. Revista Brasileira de Paleontologia, 4:51-62.

Porpino, K.O.; Fernicola, J. \& Bergqvist, L.P. 2010. Revisiting the intertropical Brazilian species Hoplophorus euphractus (Cingulata, Glyptodontoidea) and the phylogenetic affinities of Hoplophorus. Journal of Vertebrate Paleontology, 30:911-927. doi:10.1080/02724631003765735

Porpino, K.O.; Fernicola, J.C.; Cruz, L.E.; Bergqvist, L.P. 2014. The intertropical Brazilian species of Panochthus (Xenarthra, Cingulata, Glyptodontoidea): a reappraisal of their taxonomy and phylogenetic affinities. Journal of Vertebrate Paleontology, 34:1165-1179. doi:10.1080/02724634.2014.863203

Scillato-Yané, G.J.; Carlini, A.A.; Tonni, E.P. \& Noriega, J.I. 2005. Paleobiogeography of the late Pleistocene pampatheres of South America. Journal of South American Earth Sciences, 20:131-138. doi:10.1016/j.jsames.2005.06.012

Soibelzon, E.; Zurita, A. \& Carlini, A.A. 2006. Glyptodon munizi Ameghino (Mammalia, Cingulata, Glyptodontidae): redescripción y anatomia. Ameghiniana, 43:377-384.
Winge, H. 1915. Jordfundne og nulevende Gumlere (Edentata) fra Lagoa Santa, Minas Gerais, Brasilien. M'ed udsigt over gumlernes indbyrdes slaegtskab. E Museo Lundii, 1:106-139.

Woodburne, M.O. 2010. The Great American Biotic Interchange: dispersals, tectonics, climate, sea level and holding pens. Journal of Mammalia Evolution, 17:245-264. doi:10.1007/ s10914-010-9144-8

Zurita, A.E.; Miño-Boilini, A.R.; Francia, A. \& Mosquera-Arenas, J.E. 2012. The Pleistocene Glyptodontidae Gray, 1869 (Xenarthra: Cingulata) of Colombia and some considerations about the South American Glyptodontinae. Revista Brasileira de Paleontologia, 15:273-280. doi:10.4072/rbp.2012.3.04

Zurita, A.E.; Oliveira, E.V; Toriño, P.; Roriguez-Bualó, S.M.; Scillato-Yané, G.J.; Luna, C. \& Krapovickas, J. 2011. On the taxonomic status of some Glyptodontidae (Mammalia, Xenarthra, Cingulata) from the Pleistocene of South America. Annales de Paleontologie, 97:63-83. doi:10.1016/j.annpal.2011.07.003

Received in 05 September, 2017; Accepted in 18 January, 2018. 